journal club

\title{
Eine sinnvolle Neuentwicklung
}

Fragestellung: Wie wirksam und verträglich ist das neue Antikonvulsivum Brivaracetam (BRV) wirklich in der Zusatztherapie therapierefraktärer fokaler Epilepsien?

Hintergrund: BRV ist ein in Anlehnung an die chemische Struktur von Levetiracetam designtes, auf höhere Effektivität ausgelegtes neues Antikonvulsivum. Die erwartete, zu Levetiracetam (LEV) gesteigerte Wirkpotenz beruht auf der Tatsache, dass es eine höhere Bindungsaffinität an das synaptische Vesikelprotein 2A hat, bei gleich hoher Selektivität des Rezeptors. Zudem blockiert es - anders als LEV - spannungsabhängige Natriumkanäle, was ein zusätzliches Wirksamkeitspotenzial birgt. BRV ist bereits in fokalen als auch in generalisierten Epilepsiemodellen überzeugend und vielversprechend gewesen. Die EU- und FDA-Zulassung als Add-on-Therapie für fokale Epilepsien erfolgte im Februar 2016.

Patienten und Methodik: Es handelt sich um eine Metaanalyse bislang publizierter randomisierter, einfach- und doppelblinder placebokontrollierter Studien zur Add-on-Therapie mit BRV bei Erwachsenen (älter als 16 Jahre), die an einer therapierefraktären fokalen Epilepsie leiden. Im

Lattanzi S, Cagnetti C, Foschi $\mathrm{N}$ et al. Brivaracetam add-on for refractory focal epilepsy. A systematic review and meta-analysis. Neurology 2016; 86: $1344-52$ Minimum sollten die Patienten über sieben Wochen BRV erhalten haben. Die Wirksamkeit wurde anhand einer Anfallsreduktion von $\geq 50 \%$ im Vergleich zur vorliegenden Baseline erfasst. Des Weiteren erfolgte eine gesonderte Datenerhebung bezüglich beklagter $\mathrm{Ne}$ benwirkungen, inklusive der Meldung von Adverse Events, oder solchen, die Labor- oder EKG-Veränderungen mit sich brachten.

Ergebnisse: Insgesamt konnten sechs von ursprünglich 126 erfassten Studien aufgearbeitet und damit 1.715 BRV- und 684 Placebopatienten ausgewertet werden. Die Erhebung der statistischen Größe des relativen Risikos (RR) für 50 \%ige Anfallsreduktion oder sogar Anfallsfreiheit lag mit BRV bei 1,75 und war damit deutlich besser als in der Placebogruppe $(4,74)$. Wirksam waren Dosierungen ab $20 \mathrm{mg} / \mathrm{Tag}$. Die wirksamste BRV-Dosis im Hinblick auf Anfallsfreiheit lag allerdings bei $100 \mathrm{mg} / \mathrm{Tag}$, worunter sich eine siebenfach höhere Wirksamkeit im Vergleich zu Placebo darstellen ließ. In einer Subanalyse zeigte sich eine geringere Wirksamkeit von BRV bei den Patienten, die bereits LEV einnahmen, im Vergleich zu denen die LEV-naiv waren. Bei LEV-naiven Patienten reichte bereits eine BRV-Dosis von 50 mg/Tag zur $\geq 50 \%$ igen Anfallsreduktion aus. Typischerweise beklagte Nebenwirkungen waren Gereiztheit, Müdigkeit, Schläfrigkeit, Kopfschmerzen und Schwindel. Außergewöhnliches oder Unerwartetes kam nicht vor.

Schlussfolgerungen: Add-on verabreichtes BRV ist bei fokalen Epilepsien ein wirksames und ausgesprochen verträgliches Antikonvulsivum, das zudem einfach in der Handhabung ist. In Kombination mit LEV ist es aufgrund des ähnlichen chemischen Wirkansatzes etwas weniger effektiv, eignet sich aber idealerweise für Patienten, die auf LEV gut respondiert haben, es aber aufgrund von Nebenwirkungen wieder absetzen mussten.

\section{- Kommentar von Vivien Homberg, Bad Berka}

\section{Eine Option, an die unbedingt gedacht werden muss!}

Die Schwächen solcher Studien sind bekannt und müssen nicht nochmals erörtert werden. Trotz allem zeichnet sich auch hier ab, dass BRV in den Folgestudien bezüglich seiner Wirksamkeit nah an den Zulassungsdaten liegt, mit damals publizierter $55,8 \%$ Responderrate ( $\geq 50 \%$ Anfallsreduktion) im Vergleich zu $16,7 \%$ unter Placebo. Auch enttäuscht es in den Folgestudien nicht, was die gute Verträglichkeit betrifft. Hinweise auf bislang noch nicht bekannte Nebenwirkungen ergaben sich in keiner der aufbereiteten Studien. Damit bleibt festzuhalten, dass wir seit Anfang diesen Jahres ein wirksames, interaktions- sowie nebenwirkungsarmes Antikonvulsivum zur Hand haben, was hoffentlich in den Langzeitdaten weiterhin so effektiv sein wird. Dazu kommt der Komfort einer ausgesprochen einfachen Handhabung (umgehender Einsatz einer voll wirksamen Dosis) und vielfältiger Zubereitungsformen von Tablette über Saft bis hin zur i. v.-Version. Sollte es tatsächlich gelungen sein, eine „Turbo-Version“ des LEV designt zu haben, würde die Epilepsietherapie eines Tages um vieles einfacher werden. Besonders spannend scheint in diesem Kontext, dass BRV möglicherwei- se auch ein hochpotentes Antikonvulsivum bei idiopathischen Epilepsien ist. So konnte bereits ein dosisabhängiger Effekt bei photosensiblen Patienten nachgewiesen werden, wobei die pathologische Photoreaktion nicht nur relevant reduziert, sondern ganz unterdrückt wurde. Vielleicht ist es jetzt schon ein Versuch im Off-Label-Bereich wert, wenn alles andere nicht vertragen wird oder bei diesen sonst einfach zu behandelnden Epilepsien keine Wirksamkeit zeigt.

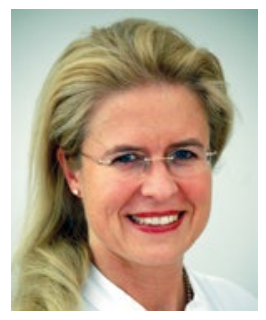

Dr. med. Vivien Homberg, Bad Berka

Chefärztin der Klinik für Neurologie, Zentralklinikum Bad Berka

E-Mail: Vivien.Homberg@zentralklinik.de 